\title{
A New Generalized Lomax Model: Statistical Properties And Applications
}

\author{
Mohamed Ibrahim ${ }^{1 *}$ and Haitham M. Yousof ${ }^{2}$ \\ ${ }^{1}$ Department of Applied Statistics and Insurance, Faculty of Commerce, Damietta University, Egypt. \\ ${ }^{2}$ Department of Statistics, Mathematics and Insurance, Benha University, Egypt.
}

\begin{abstract}
In this paper, a new version of the Poisson Lomax distributions is proposed and studied. The new density is expressed as a linear mixture of the Lomax densities. The failure rate function of the new model can be increasing-constant, increasing, $U$ shape, decreasing and upside down-increasing. The statistical properties are derived and four applications are provided to illustrate the importance of the new density. The method of maximum likelihood is used to estimate the unknown parameters of the new density. Adequate fitting is provided by the new model.
\end{abstract}

Keywords: Truncated Poisson Distribution; Lomax Distribution; Moments, Moment Generating Function; Maximum Likelihood; Simulation; Modelling Real Data.

\footnotetext{
*mohamed_ibrahim@du.edu.eg
} 


\section{Introduction and physical motivation}

The cumulative distribution function (CDF) of the two parameters generalized Lomax distribution (GL) is given as

$$
G_{\theta, \gamma}(y)=\left[1-(y+1)^{-\gamma}\right]^{\theta} \forall y>0
$$

where $\theta>0$ and $\gamma>0$ are the shape parameters. The corresponding probability density function (PDF) is given by

$$
g_{\theta, \gamma}(y)=\theta \gamma(y+1)^{-1-\gamma}\left[1-(y+1)^{-\gamma}\right]^{\theta-1} \forall y>0
$$

For $\theta=1$, we have the one parameter Lomax (L) (or Pareto type II (PaII)) model. Clearly, the GL model is a special case from the generalized Burr XII (GBXII) model. Following Yousof et al. (2017), we derive a new model called the Burr X generalized Lomax (BXGL) model defined by the CDF given by

$$
\boldsymbol{W}_{\delta, \theta, \gamma}(y)=\left[1-\exp \left(-\left\{\left[1-(y+1)^{-\gamma}\right]^{-\theta}-1\right\}^{-2}\right)\right]^{\delta} \forall y>0
$$

where $\delta>0$ is refer to shape parameter. Assume having a system of $N$ independent functioning subsystems at a given time where $N$ has zero truncated Poisson (ZTP) distribution with parameter $a$. The probability mass function (PMF) of $N$ is formulated as follows

$$
\left.p_{Z T P}^{(a)}(N=n)\right|_{\left(a \in \boldsymbol{R}^{+}-\{0\} \text { and } n=1,2, \ldots\right)}=\left[\exp (-a) a^{n}\right] /\left(n ! \boldsymbol{v}_{(a)}\right) .
$$

Note that for ZTP random variable (r.v.), the expected value $\boldsymbol{E}(N \mid a)$ and the variance $\operatorname{Var}(N \mid a)$ are, respectively, given by

$$
\boldsymbol{E}(N \mid a)=\frac{a}{\boldsymbol{v}_{(a)}}
$$

and

$$
\operatorname{Var}(N \mid a)=\frac{a+a^{2}}{\boldsymbol{v}_{(a)}}-\frac{a^{2}}{\left[\boldsymbol{v}_{(a)}\right]^{2}}
$$

where $\boldsymbol{v}_{(a)}=-\exp (-a)+1$. Suppose that the failure time of each subsystem has the BXGL.

Let $Z_{i}$ denotes the failure time of the $i^{\text {th }}$ subsystem and let

Then the conditional CDF of $Y$ given $N$ is

$$
Y=\min \left\{Z_{1}, Z_{2}, \cdots, Z_{N}\right\} \text {. }
$$

$$
F(y \mid N)=1-\operatorname{Pr}(Y>y \mid N)=1-\left[1-\boldsymbol{W}_{\delta, \theta, \gamma}(y)\right]^{N} .
$$

Therefore, the unconditional CDF of the Poisson Burr X generalized Lomax (PBXGL) CDF can be expressed as

$$
\begin{gathered}
\left.F_{\underline{\Psi}}(y)\right|_{(a \in \boldsymbol{R}-\{0\})} \\
=\boldsymbol{v}_{(a)}\left(1-\exp \left\{-a\left[1-\exp \left(-\left\{\left[1-(y+1)^{-\gamma}\right]^{-\theta}-1\right\}^{-2}\right)\right]^{\delta}\right\}\right),
\end{gathered}
$$

where $\underline{\boldsymbol{\Psi}}=(a, \delta, \theta, \gamma)$ refer to the parameters vector. The corresponding PDF is 


$$
\begin{gathered}
\left.f_{\underline{\Psi}}(y)\right|_{(a \in \boldsymbol{R}-\{0\})}=2 a \delta \theta \gamma \frac{(y+1)^{-1-\gamma}\left[1-(y+1)^{-\gamma}\right]^{2 \theta-1}}{\boldsymbol{v}_{(a)}\left\{1-\left[1-(y+1)^{-\gamma}\right]^{\theta}\right\}^{3}} \\
\times \frac{\exp \left(-\left\{\left[1-(y+1)^{-\gamma}\right]^{-\theta}-1\right\}^{-2}\right)}{\left[1-\exp \left(-\left\{\left[1-(y+1)^{-\gamma}\right]^{-\theta}-1\right\}^{-2}\right)\right]^{1-\delta}} \\
\times \exp \left\{-a\left[1-\exp \left(-\left\{\left[1-(y+1)^{-\gamma}\right]^{-\theta}-1\right\}^{-2}\right)\right]^{\delta}\right\} .
\end{gathered}
$$

The hazard rate function (HRF) can be calculated by $f_{\underline{\Psi}}(y) /\left[1-F_{\underline{\Psi}}(y)\right]$. The PBXGL density can be right skewed and unimodal (see Figure 1) whereas the PBXGL HRF can be increasing, $\mathbf{J}$ shape, $\mathbf{U}$ shape, decreasing or upside down (see Figure 2). We are also motivated to introduce the PBXGL distribution that contains many new lifetime models as illustrated in Table 1
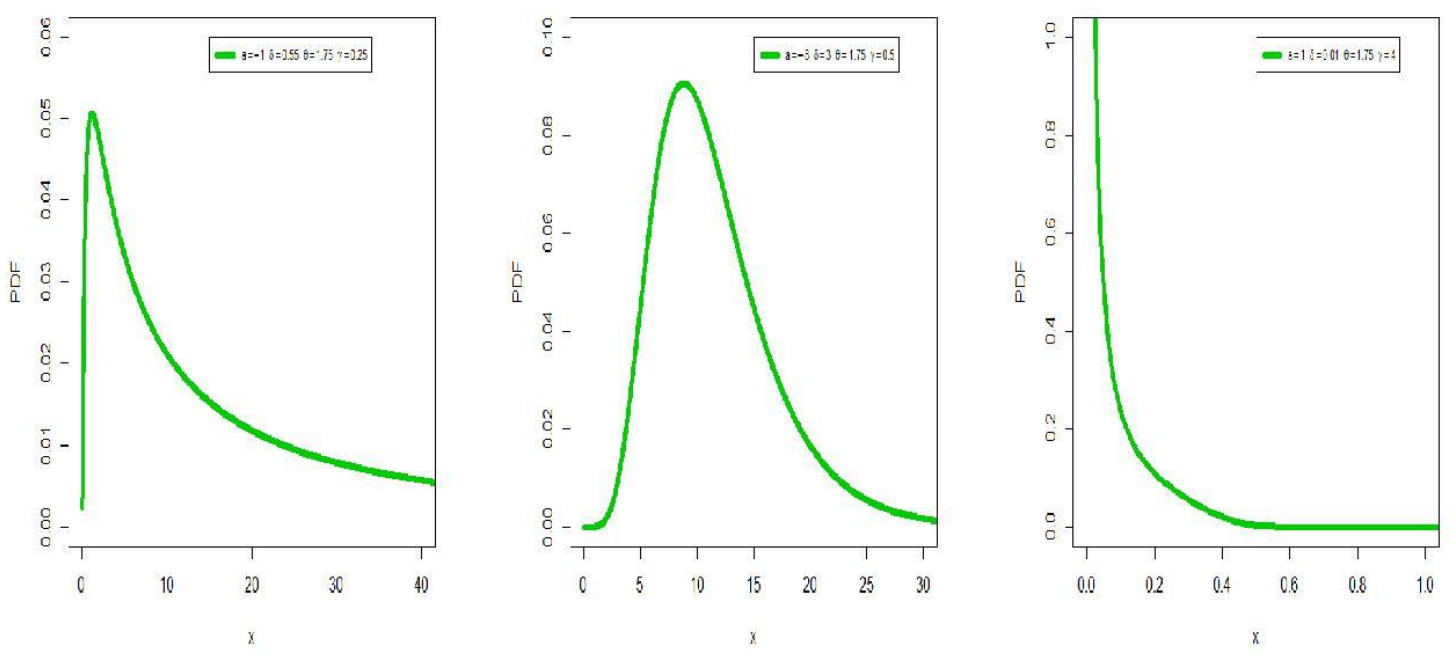

Figure 1: Plots of the PBXGL PDF at some parameter values. 

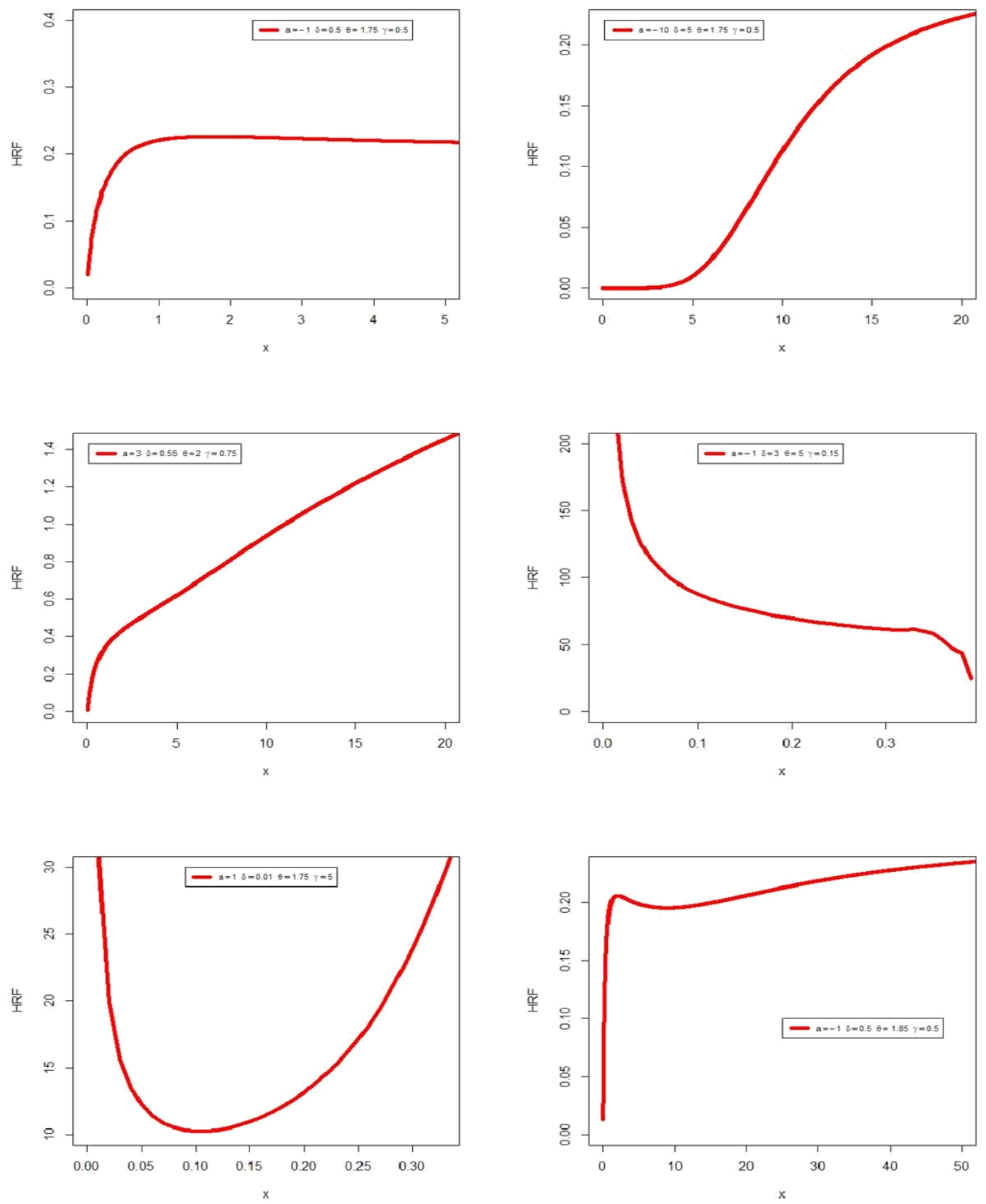

Figure 2: Plots of the PBXGL HRF at some parameter values. 
Table 1: CDF for sub-models of the PBXGL model.

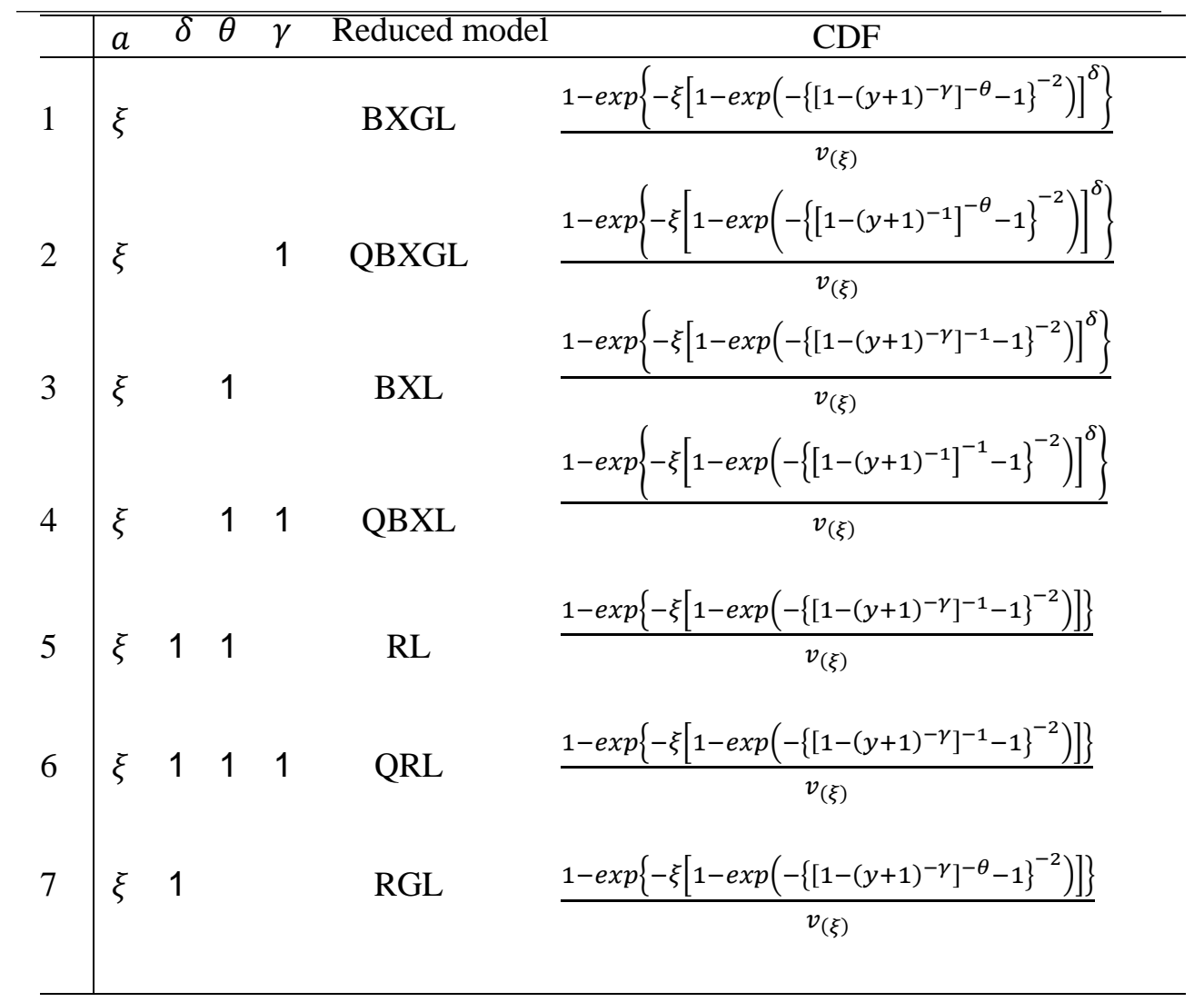




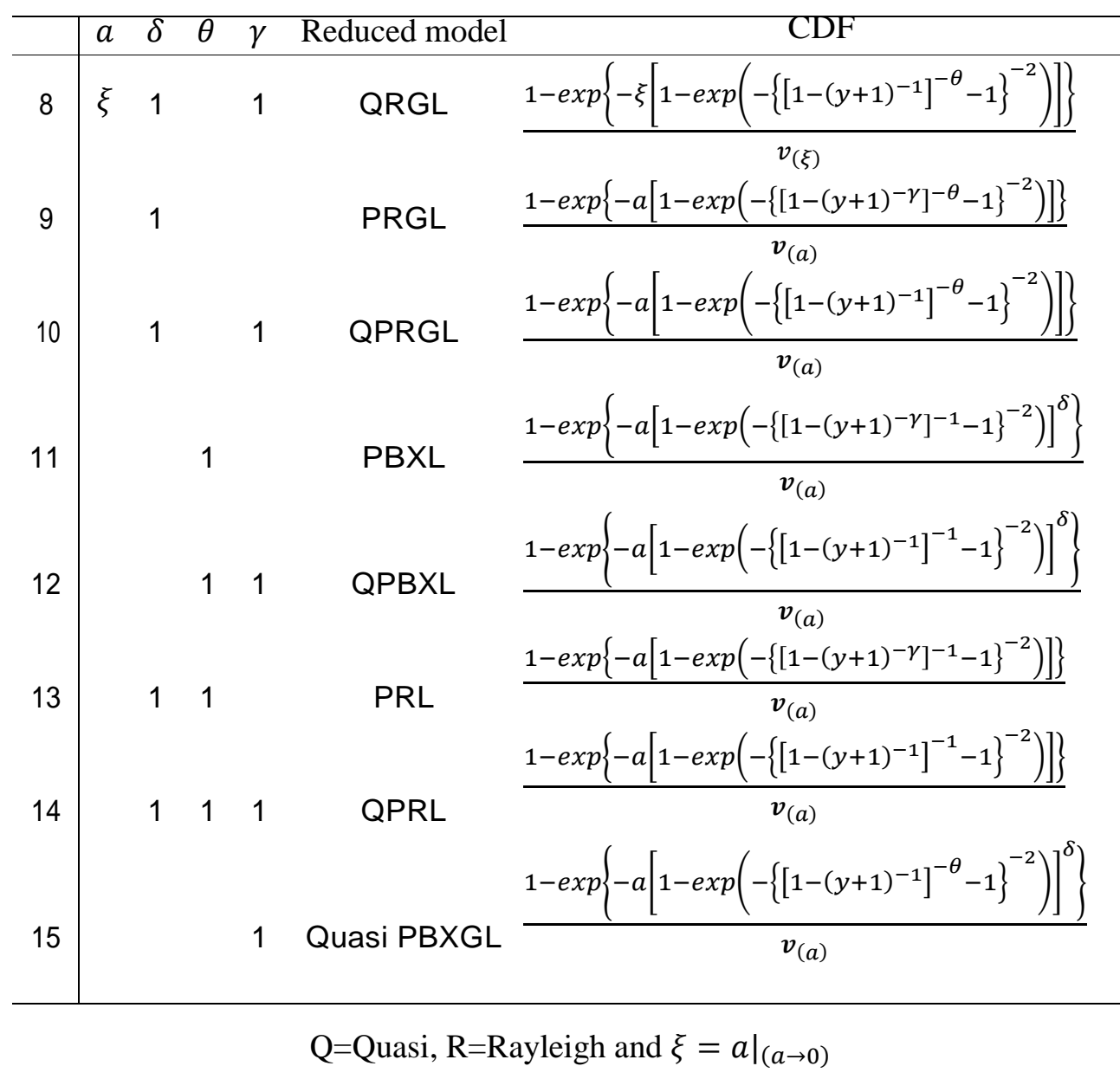

This work is organized as follows: In Section 2, we derive some new properties of the PBXGL model. The maximum likelihood method is addressed in Section 3. Simulation studies are presented in Section 4. In Section 5, the potentiality of the proposed model is illustrated using three real data sets. Section 6 gives some concluding remarks.

\section{Mathematical properties}

\subsection{Useful expansions}

Using the power series

the $f_{\underline{\Psi}}(y)$ in $(6)$ can be written as

$$
\exp \left(\zeta_{1}\right)=\sum_{\zeta_{2}=0}^{\infty} \frac{\zeta_{1}^{\zeta_{2}}}{\zeta_{2} !}
$$

$$
\begin{gathered}
f_{\underline{\Psi}}(y)=\sum_{\tau=0}^{\infty} \frac{2 a^{1+\tau} \delta \theta \gamma}{\boldsymbol{v}_{(a)}}(-1)^{\tau} \\
\times \exp \left(-\left\{\left[1-(y+1)^{-\gamma} \%\right]^{-\theta}-1\right\}^{-2}\right) \\
\times \frac{(y+1)^{-1-\gamma}\left[1-(y+1)^{-\gamma}\right]^{2 \theta-1}}{\left\{1-\left[1-(y+1)^{-\gamma}\right]^{\theta}\right\}^{3}}
\end{gathered}
$$




$$
\times\left\{1-\exp \left(-\left\{\left[1-(y+1)^{-\gamma}\right]^{-\theta}-1\right\}^{-2}\right)\right\}^{\delta(\tau+1)-1}
$$

Consider the following power series

$$
\left(1-\zeta_{1}\right)^{\zeta_{2}}=\left.\sum_{m=0}^{\infty} \frac{(-1)^{m} \Gamma\left(1+\zeta_{2}\right)}{m ! \Gamma\left(1+\zeta_{2}-m\right)} \zeta_{1}^{m}\right|_{\left|\zeta_{1}\right|<1 \text { and } \zeta_{2}>0 \text { is a real non-integer }}
$$

Applying (8) to (7) we have

$$
\begin{aligned}
& f_{\Psi}(y)=\frac{2 \delta \gamma(y+1)^{-1-\gamma}\left[1-(y+1)^{-\gamma}\right]^{2 \theta-1}}{\boldsymbol{v}_{(a)}\left\{1-\left[1-(y+1)^{-\gamma}\right]^{\theta}\right\}^{3}} \\
& \times \sum_{\tau, i=0}^{\infty} \frac{a^{1+\tau}(-1)^{\tau+i} \Gamma(\delta(\tau+1))}{i ! \Gamma(\delta(\tau+1)-i)} \\
& \exp \left(-(i+1)\left\{\left[1-(y+1)^{-\gamma}\right]^{-\theta}-1\right\}^{-2}\right) .
\end{aligned}
$$

Applying the power series to the term

then, equation (9) becomes

$$
\exp \left(-(i+1)\left\{\left[1-(y+1)^{-\gamma}\right]^{-\theta}-1\right\}^{-2}\right)
$$

$$
\begin{aligned}
& f_{\underline{\Psi}}(y)=\sum_{\tau, i, d=0}^{\infty} \frac{2 \delta a^{1+\tau}(-1)^{\tau+i+d}(i+1)^{d} \Gamma(\delta(\tau+1))}{i ! d ! \boldsymbol{v}_{(a)} \Gamma(\delta(\tau+1)-i)} \\
& \times \frac{\theta \gamma(y+1)^{-1-\gamma}\left[1-(y+1)^{-\gamma}\right]^{2 \theta(d+1)-1}}{\left\{1-\left[1-(y+1)^{-\gamma}\right]^{\theta}\right\}^{3+2 d}}
\end{aligned}
$$

Consider the series expansion

$$
\left(1-\zeta_{1}\right)^{-\zeta_{2}}=\left.\sum_{\omega=0}^{\infty} \frac{\Gamma\left(\zeta_{2}+\omega\right)}{\omega ! \Gamma\left(\zeta_{2}\right)} \zeta_{1}^{\omega}\right|_{\left(\left|\zeta_{1}\right|<1, \zeta_{2}>0\right)},
$$

and, applying (11) to (10) for the term

equation (10) can be written as

$$
\left\{1-\left[1-(y+1)^{-\gamma}\right]^{\theta}\right\}^{-(3+2 d)}
$$

$$
\begin{aligned}
f_{\underline{\boldsymbol{\Psi}}}(y)=\sum_{\substack{\tau, i, d, \kappa=0\\
}}^{\infty} \frac{2 \delta a^{1+\tau}(-1)^{\tau+i+d}(i+1)^{d}}{i ! d ! \kappa ! \boldsymbol{v}_{(a)}} \frac{\Gamma(\delta(\tau+1)) \Gamma(3+2 d+\kappa)}{\Gamma(\delta(\tau+1)-i) \Gamma(2 d+3)} \\
\\
\quad \times \theta \gamma(y+1)^{-1-\gamma}\left[1-(y+1)^{-\gamma}\right]^{[2(d+1)+\kappa] \theta-1}
\end{aligned}
$$

which can be expressed as

where

$$
f_{\underline{\Psi}}(y)=\sum_{r=0}^{\infty} c_{r} g_{\gamma(1+r)}(y)
$$

and

$$
\begin{gathered}
c_{r}=\frac{(-1)^{r} \Gamma([2(1+d)+\kappa] \theta)}{r ! \Gamma([2(1+d)+\kappa] \theta-r)} \\
\times \sum_{d, \kappa=0}^{\infty} \frac{2 \delta a^{1+\tau}(-1)^{d} \Gamma(3+2 d+\kappa)}{d ! \kappa ! v_{(a)} \Gamma(2 d+3)} \\
\times \sum_{\tau, i=0}^{\infty} \frac{(-1)^{\tau+i} \Gamma(\delta(\tau+1))(i+1)^{d}}{i ! \Gamma(\delta(\tau+1)-i)}
\end{gathered}
$$

$$
g_{\gamma(1+r)}(y)=\gamma(1+r)(y+1)^{-1-\gamma(1+r)} \text {, }
$$

is the PDF of the Lomax model with parameter $\gamma(1+r)$. Equation (12) reveals that the density of the PBXGL model can be expressed as a linear mixture of the Lomax densities. Thus, several mathematical properties of the PBXGL model can be obtained from the Lomax densities. Simi- 
larly, the CDF of the PBXGL model can also be expressed as a mixture of the Lomax CDFs and given by

$$
F_{\underline{\Psi}}(y)=\sum_{r=0}^{\infty} c_{r} G_{\gamma(1+r)}(y)
$$

where

$$
G_{\gamma(1+r)}(y)=1-(y+1)^{-\gamma(1+r)}
$$

is the CDF of the Lomax model with parameters $\gamma(1+r)$.

\subsection{Quantile and random number generation}

The quantile function $(\mathrm{QF})$ of $Y$; where $Y \sim P B X G L(a, \delta, \theta, \gamma)$,is obtained by inverting (5) as

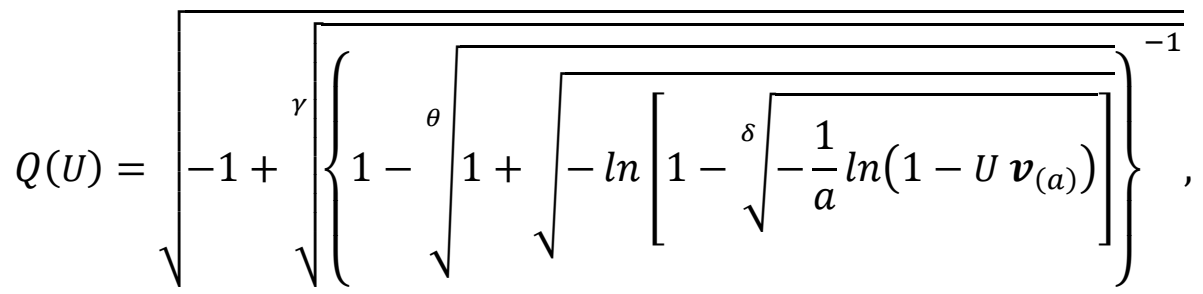

simulating the PBXGL r.v. is straightforward. If $U$ is a uniform variate on the unit interval $(0,1)$, then the r.v. $y=Q(U)$ follows $(5)$.

\subsection{Ordinary moments}

The $n^{\text {th }}$ ordinary moment of $Y$, say $\mu_{n}^{\prime}$, follows from (12) as

$$
\mu_{n}^{\prime}=\boldsymbol{E}\left(Y^{n}\right)=\left.\sum_{r=0}^{\infty} c_{r}(1+r) \gamma B((1+r) \gamma-1, n+1)\right|_{[n<\gamma(1+r)]},
$$

where

$$
B\left(\zeta_{1}, \zeta_{2}\right)=\int_{0}^{\infty} \omega^{\zeta_{1}-1}(1+\omega)^{-\left(\zeta_{1}+\zeta_{2}\right)} d \omega,
$$

setting $n=1$ in (14) gives the mean of $Y$ as

$$
\mu_{1}^{\prime}=\boldsymbol{E}(Y)=\left.\sum_{r=0}^{\infty} c_{r}(1+r) \gamma B((1+r) \gamma-1,2)\right|_{[1<\gamma(1+r)]}
$$

\subsection{Incomplete moment}

The $n^{\text {th }}$ incomplete moment of $Y$ is defined by

$$
m_{n}(t)=\int_{-\infty}^{t} y^{n} f(y) d y
$$

Based on (12), the $n^{\text {th }}$ incomplete moment of $Y$ becomes

where

$$
m_{n}(t)=\left.\sum_{r=0}^{\infty} c_{r}(1+r) \gamma B(t ;(1+r) \gamma-1, n+1)\right|_{[n<\gamma(1+r)]},
$$

$$
B\left(\cdot ; \zeta_{1}, \zeta_{2}\right)=\int_{0}^{\cdot} \omega^{\zeta_{1}-1}(1+\omega)^{-\left(\zeta_{1}+\zeta_{2}\right)} d \omega
$$

is incomplete beta functions of the second type, respectively. 


\subsection{Moment generating function (MGF)}

The MGF of $Y$, say $M_{Y}(t)=\boldsymbol{E}[\exp (t Y)]$, can be obtained via (12) as

$$
M_{Y}(t ; a, \delta, \theta, \gamma)=\left.\sum_{r, n=0}^{\infty} \frac{t^{n}}{\bar{n} !} c_{r}(1+r) \gamma B((1+r) \gamma-1, n+1)\right|_{[n<\gamma(1+r)]} .
$$

\section{Parameter estimation}

Consider the estimation of the unknown parameters $(a, \delta, \theta, \gamma)$ of the PBXGL model from the complete samples by maximum likelihood (ML) method. Suppose that $Y_{1}, \cdots, Y_{n}$ be a random sample from the PBXGL model with parameter vector $\underline{\boldsymbol{\Psi}}=(a, \delta, \theta, \gamma)^{\top}$. The $\log$ likelihood function $\left(\ell_{n}(\underline{\boldsymbol{\Psi}})\right.$ ) for $\underline{\boldsymbol{\Psi}}$ is given by

$$
\begin{aligned}
\ell_{n}(\underline{\boldsymbol{\Psi}})= & n \log 2+n \log \delta+n \log a+n \log \gamma-n \log \left[\boldsymbol{v}_{(a)}\right]-(\gamma+1) \sum_{i=1}^{n} \log \left(1+y_{i}\right) \\
& +(2 \theta-1) \sum_{i=1}^{n} \log \left[1-\left(1+y_{i}\right)^{-\gamma}\right]-3 \sum_{i=1}^{n} \log \left(1-q_{i}\right) \\
& -\sum_{i=1}^{n} m_{i}+(\delta-1)-\sum_{i=1}^{n} \log \left[1-\exp \left(-m_{i}\right)\right]-a \sum_{i=1}^{n}\left[1-\exp \left(-m_{i}\right)\right]^{\delta},
\end{aligned}
$$

where

$$
q_{i}=\left[1-\left(1+y_{i}\right)^{-\gamma}\right]^{\theta} \text { and } m_{i}=\left(\frac{q_{i}}{1-q_{i}}\right)^{2} .
$$

The function $\ell_{n}(\underline{\Psi})$ can be maximized numerically via SAS (PROC NLMIXED) or R (optim) or (Ox) program (via sub-routine MaxBFGS). The components of the score vector

can easily be derived.

$$
\boldsymbol{U}(\underline{\boldsymbol{\Psi}})=\frac{\partial \ell}{\partial \underline{\boldsymbol{\Psi}}}=\left(\frac{\partial \ell_{n}(\underline{\boldsymbol{\Psi}})}{\partial a}, \frac{\partial \ell_{n}(\underline{\boldsymbol{\Psi}})}{\partial \delta}, \frac{\partial \ell_{n}(\underline{\boldsymbol{\Psi}})}{\partial \theta}, \frac{\partial \ell_{n}(\underline{\boldsymbol{\Psi}})}{\partial \gamma}\right)^{\top}
$$

\section{Simulation study}

Using (13), we simulate the PBXGL model by taking $n=20,50,150,500$ and 1000 . For each sample size, we evaluate the ML estimations (MLEs) of the parameters using the optim function of the Mathcad software. Then, we repeat this process 1000 times and compute the averages of the estimates (AEs) and mean squared errors (MSEs). Table 2 gives the simulation results. The values in Table 2 indicate that the MSEs of $\hat{a}, \hat{\delta}, \hat{\theta}$ and $\hat{\gamma}$ decay toward zero when $n$ increases for all settings of $a, \delta, \theta$ and $\gamma$ as expected under first-under asymptotic theory. The AEs of $(\mathbf{I}: a=0.5, \delta=1.5, \theta=0.9$ and $\gamma=1$. and $\mathbf{I I}: a=2.5, \delta=0.8, \theta=1.6$ and $\gamma=0.6$ when $n$ increases. This fact supports that the asymptotic normal distribution provides an adequate approximation to the finite sample distribution of the MLEs. 
Table 2: The AEs, biases and MSEs based on 1000 simulations.

\begin{tabular}{|c|c|c|c|c|c|c|}
\hline $\mathrm{n}$ & $\Theta$ & AEs & MSE & $\Theta$ & AEs & MSE \\
\hline & $I$ & & & II & & \\
\hline \multirow[t]{4}{*}{20} & $a$ & 0.550554 & 0.687748 & $a$ & 2.633217 & 1.137718 \\
\hline & $\delta$ & 1.535720 & 0.161429 & $\delta$ & 0.813639 & 0.019832 \\
\hline & $\theta$ & 0.896758 & 0.004083 & $\theta$ & 1.606293 & 0.026339 \\
\hline & $\gamma$ & 1.212598 & 0.008217 & $\gamma$ & 0.605537 & 0.004376 \\
\hline \multirow[t]{4}{*}{50} & $a$ & 0.479577 & 0.275757 & $a$ & 2.502158 & 0.409679 \\
\hline & $\delta$ & 1.533873 & 0.064385 & $\delta$ & 0.812062 & 0.007323 \\
\hline & $\theta$ & 0.901872 & 0.001714 & $\theta$ & 1.610576 & 0.010553 \\
\hline & $\gamma$ & 1.200769 & 0.003230 & $\gamma$ & 0.599028 & 0.001679 \\
\hline \multirow[t]{4}{*}{150} & $a$ & 0.495784 & 0.086247 & $a$ & 2.489394 & 0.134721 \\
\hline & $\delta$ & 1.509371 & 0.019408 & $\delta$ & 0.805320 & 0.002347 \\
\hline & $\theta$ & 0.900356 & 0.000549 & $\theta$ & 1.605307 & 0.003486 \\
\hline & $\gamma$ & 1.200597 & 0.001031 & $\gamma$ & 0.598894 & 0.000561 \\
\hline \multirow[t]{4}{*}{500} & $a$ & 0.494325 & 0.025012 & $a$ & 2.511130 & 0.041126 \\
\hline & $\delta$ & 1.505033 & 0.005616 & $\delta$ & 0.799685 & 0.000695 \\
\hline & $\theta$ & 0.900495 & 0.000161 & $\theta$ & 1.599278 & 0.001054 \\
\hline & $\gamma$ & 1.199632 & 0.000299 & $\gamma$ & 0.600599 & 0.000173 \\
\hline \multirow[t]{4}{*}{1000} & $a$ & 0.499884 & 0.012859 & $a$ & 2.493011 & 0.019928 \\
\hline & $\delta$ & 1.501229 & 0.002891 & $\delta$ & 0.800670 & 0.000348 \\
\hline & $\theta$ & 0.900020 & 0.000083 & $\theta$ & 1.600639 & 0.000526 \\
\hline & $\gamma$ & 1.200135 & 0.000154 & $\gamma$ & 0.599901 & 0.000085 \\
\hline
\end{tabular}

\section{Modeling real data}

Four real data sets are provided to illustrate the importance, potentiality and flexibility of the PBXGL model. According to these data, we compare the PBXGL distribution with BXII, Marshall-Olkin BXII (MOBXII), Topp Leone BXII (TLBXII), Zografos-Balakrishnan BXII (ZB-BXII), Five Parameters beta BXII (FBBXII), BBXII, B exponentiated BXII (BEBXII), Five Parameters Kumaraswamy BXII (FKwBXII) and KwBXII distributions given in Brito et al. (2017), Hamedani et al. (2017), Hamedani et al. (2018), Yousof et al. (2018), Altun et al. (2018 a), Korkmaz et al. (2018), Altun et al. (2018 b), Elbiely and Yousof (2019), Hamedani et al. (2019), Goual and Yousof (2019), Goual et al. (2019), Gad et al. (2019), Ibrahim (2019), Yousof et al. (2019 a) and Yousof et al. (2019 b).

Data set I: $\{0.98,5.56,2.83,3.68,2.00,3.51,0.85,1.61,3.28,2.95,5.08,0.39,1.57,3.19,4.90$, $2.74,2.73,2.50,3.60,3.11,2.93,2.85,2.77,2.76,1.73,2.48,3.22,3.70,3.27,2.87,1.47,3.11$, $4.42,2.81,3.15,1.92,1.84,1.22,2.17,1.61,2.12,3.09,2.97,4.20,2.35,1.41,1.59,1.12,1.69$, $2.79,1.89,1.87,3.39,3.33,2.55,3.68,3.19,1.71,1.25,4.70,2.88,3.68,1.08,3.22,3.75,2.96$, $2.55,2.59,2.97,1.57,2.17,4.38,2.03,2.82,2.53,3.31,2.38,1.36,0.81,1.17,1.84,12.40,3.15$, $2.67,3.31,2.81,2.56,2.17,4.91,1.59,1.18,2.48,2.03,1.69,2.43,3.39,3.56,0.80,2.05,3.65\}$ called breaking stress data. This data set consists of 100 observations of breaking stress of carbon fibers (in Gba) given by Nichols and Padgett (2006). 
Data set II: $\{0.1,0.33,1.08,1.08,1.08,0.44,0.56,0.59,0.72,0.74,0.77,2.54,2.78,2.93,3.27$, $3.42,0.92,0.93,0.96,1,1,1.02,1.05,1.07,07,1.09,1.12,1.13,1.15,1.36,1.39,1.44,1.83$, $1.95,1.96,1.97,2.02,1.16,1.2,1.21,1.22,1.22,1.24,1.3,1.34,2.13,1.46,1.53,1.59,1.6,1.63$, $1.63,1.68,1.71,1.72,1.76,2.15,2.16,2.22,2.3,2.31,2.4,2.45,2.51,2.53,2.54,3.47,3.61$, $4.02,4.32,4.58,5.55\}$ called survival times in days of 72 guinea pigs infected with virulent tubercle bacilli, originally observed and reported by Bjerkedal (1960).

Data set III: $\{5.9,20.4,13.3,8.5,21.6,14.9,16.2,17.2,7.8,6.1,9.2,10.2,9.6,18.5,5.1,6.7,17$, 9.2, 26.2, 21.9,16.7,21.3, 35.4, 14.3, 8.6, 9.7, 39.2, 35.7, 15.7, 9.7, 10, 4.1, 36, 8.5, 8, 8.5, 10.6, 19.1, 20.5, 7.1, 7.7,18.1, 16.5, 8.4, 11, 11.6, 11.9, 5.2, 6.8, 11.9, 7, 8.6,12.5, 10.3, 11.2, 6.1, 8.9, $7.1,10.8\}$ called taxes revenue data (in 1000 million Egyptian pounds).

Data set IV: $\{65,56,26,22,1,1,5,65,16,22,3,4,2,3,56,65,17,7,156,8,4,3,30,4,100$, $134,16,108,121,4,39,143,43\}$ called leukaemia data. This real data set gives the survival times, in weeks, of 33 patients suffering from acute Myelogeneous Leukaemia (see Feigl and Zelen (1965)).

The total time test (TTT) plots (see Aarset (1987)) for the four real data sets is presented in Figure 3. These plots indicate that the empirical HRFs of data sets I, II and III are increasing. and $U$ shape for data set IV. We consider the following goodness-of fit statistics: the Akaike Information Criterion (AIC), Bayesian Information Criterion (BIC), Hannan-Quinn Information Criterion (HQIC), consistent Akaike Information Criterion (CAIC), where

$$
\begin{gathered}
A I C=2 m_{(p)}-2 \ell(\underline{\hat{\boldsymbol{\Psi}}}), \\
B I C=m_{(p)} \log (n)-2 \ell(\underline{\hat{\boldsymbol{\Psi}}}), \\
H Q I C=2 m_{(p)} \log [\log (n)]-2 \ell(\underline{\hat{\boldsymbol{\Psi}}})
\end{gathered}
$$

and

$$
C A I C=\frac{2 n m_{(p)}}{-1+n-m_{(p)}}-2 \ell(\underline{\hat{\boldsymbol{\Psi}}}),
$$

where $m_{(p)}$ is the number of parameters, $n$ is the sample size, $\ell(\underline{\underline{\boldsymbol{\Psi}}})$ is the maximized $\log$ likelihood. Generally, the smaller statistics indicates the better fit. Tables 3, 5, 7 and 9 gives the MLEs, standard errors (SEs) and confidence interval (CIs) for the data set I, II , III and IV respectively. Based on the values in Tables 4, 6, 8 and 10 and Figures 4-8 the PBXGL model provides the best fits compared to other extensions of the BXII model for the three applications with smallest values for BIC, AIC, CAIC and HQIC. 


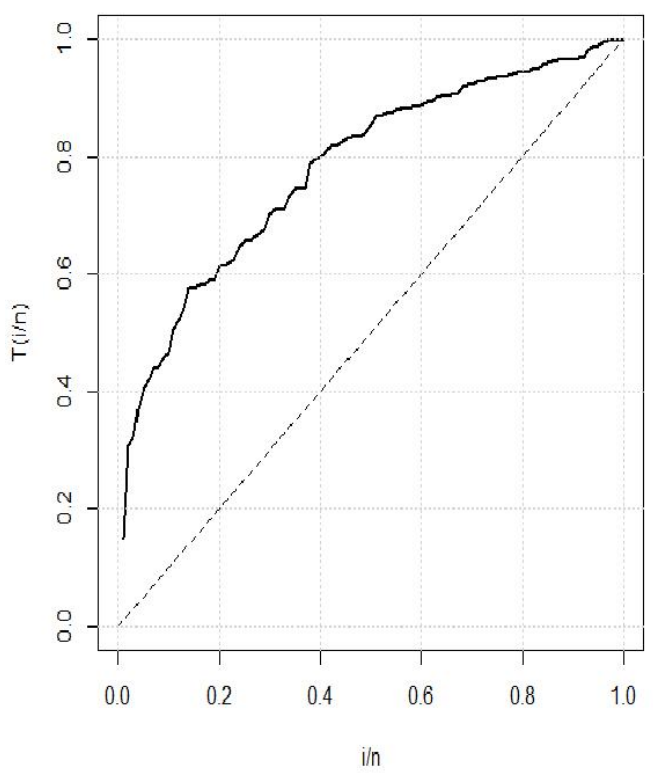

Data I

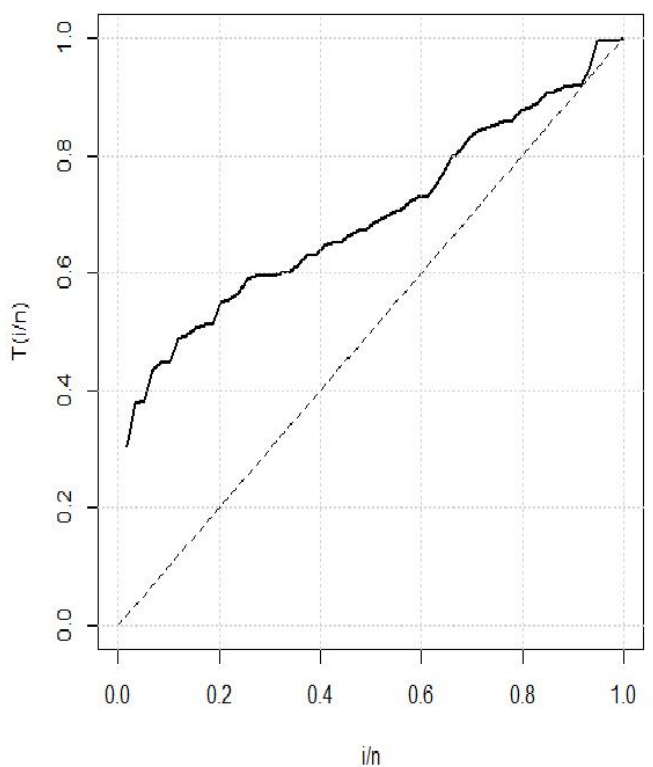

Data III

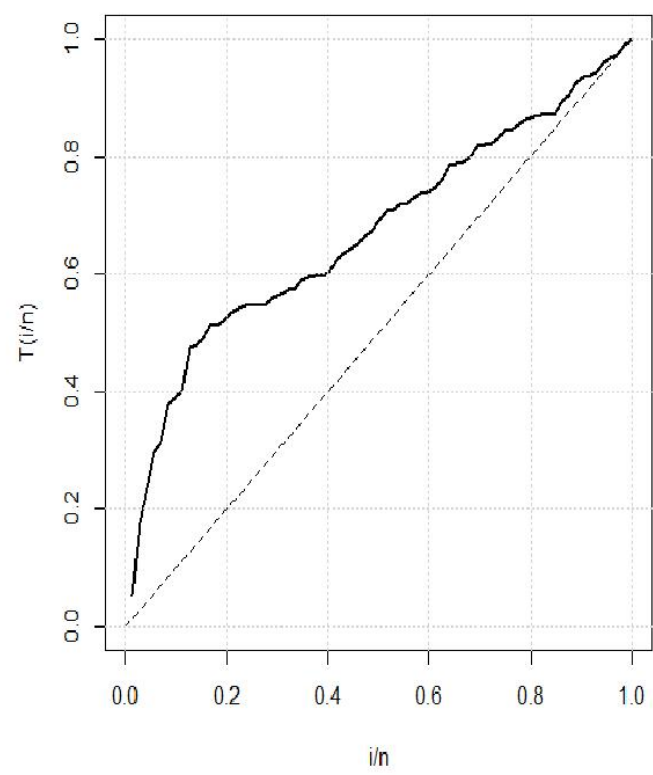

Data II

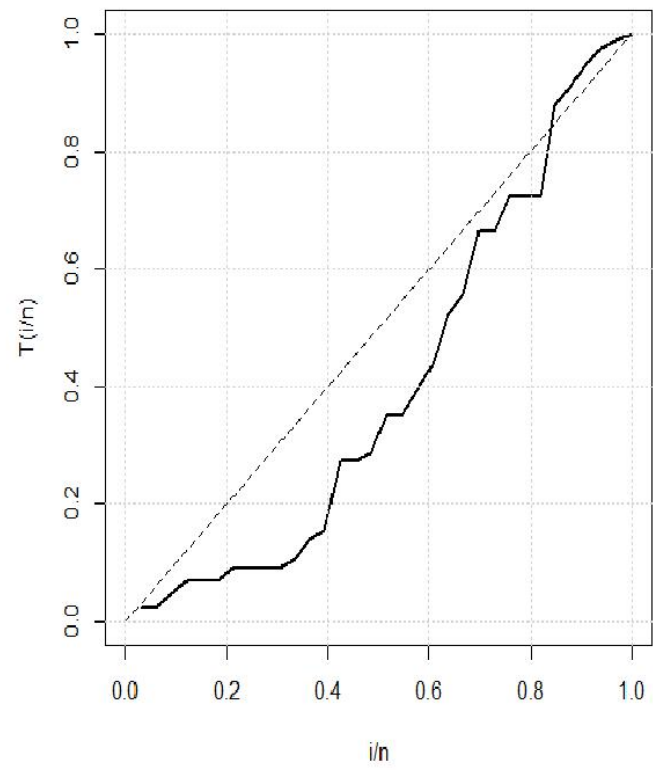

Data IV

Figure 3: TTT plots. 
Table 3: MLEs, SEs and (CIs) for the data set $\mathbf{I}$.

\begin{tabular}{|c|c|}
\hline Model & Estimates \\
\hline $\operatorname{BXII}(\alpha, \beta)$ & $\begin{array}{c}5.941,0.187 \\
(1.279),(0.044) \\
(3.43,8.45),(0.10,0.27)\end{array}$ \\
\hline $\operatorname{MOBXII}(\alpha, \beta, \gamma)$ & $\begin{array}{c}1.192,4.834,838.73 \\
(0.952),(4.896),(229.34) \\
(0,3.06),(0,14.43),(389.22,1288.24)\end{array}$ \\
\hline $\operatorname{TLBXII}(\alpha, \beta, \gamma)$ & $\begin{array}{c}1.350,1.061,13.728 \\
(0.378),(0.384),(8.400) \\
(0.61,2.09),(0.31,1.81),(0,30.19)\end{array}$ \\
\hline $\operatorname{KwBXII}(a, \delta, \alpha, \beta)$ & $\begin{array}{c}48.103,79.516,0.351,2.730 \\
(19.348),(58.186),(0.098),(1.077) \\
(10.18,86.03),(0,193.56),(0.16,0.54),(0.62,4.84)\end{array}$ \\
\hline $\operatorname{BBXII}(a, \delta, \alpha, \beta)$ & $\begin{array}{c}359.683,260.097,0.175,1.123 \\
(57.941),(132.213),(0.013),(0.243) \\
(246.1,473.2),(0.96,519.2),(0.14,0.20),(0.65,1.6)\end{array}$ \\
\hline $\operatorname{BEBXII}(a, \delta, \alpha, \beta, \gamma)$ & $\begin{array}{c}0.381,11.949,0.937,33.402,1.705 \\
(0.078),(4.635),(0.267),(6.287),(0.478) \\
(0.23,0.53),(2.86,21),(0.41,1.5),(21,45),(0.8,2.6)\end{array}$ \\
\hline $\operatorname{FBBXII}(a, \delta, \alpha, \beta, \gamma)$ & $\begin{array}{c}0.421,0.834,6.11,1.67,3.450 \\
(0.011),(0.943),(2.314),(0.226),(1.957) \\
(0.4,0.44),(0.2 .7),(1.6,10.7),(1.23,2.1),(0,7)\end{array}$ \\
\hline $\operatorname{FKwBXII}(a, \delta, \alpha, \beta, \gamma)$ & $\begin{array}{c}0.542,4.223,5.313,0.411,4.152 \\
(0.137),(1.882),(2.318),(0.497),(1.995) \\
(0.3,0.8),(0.53,7.9),(0.9,9),(0,1.7),(0.2,8)\end{array}$ \\
\hline $\operatorname{ZBBXII}(a, \alpha, \beta)$ & $\begin{array}{c}123.101,0.368,139.247 \\
(243.011),(0.343),(318.546) \\
(0,599.40),(0,1.04),(0,763.59)\end{array}$ \\
\hline $\operatorname{PBXGL}(a, \delta, \theta, \gamma)$ & $\begin{array}{c}-1.37,1.105,0.97,1.93 \\
(1.6),(0.648),(0.385),(1.551) \\
(-1.8,4.57),(0,2.3),(0.21,1.73),(0,5.03) \\
\end{array}$ \\
\hline
\end{tabular}


Table 4: AIC, BIC, CAIC and HQIC values for the data set $\mathbf{I}$.

\begin{tabular}{|c|c|}
\hline Model & AIC, BIC, CAIC, HQIC \\
\hline$\overline{\text { BXII }}$ & $\overline{382.94,388.15,383.06,385.05}$ \\
\hline MOBXII & $305.78,313.61,306.03,308.96$ \\
\hline TLBXII & $323.52,331.35,323.77,326.70$ \\
\hline KwBXII & $303.76,314.20,304.18,308.00$ \\
\hline BBXII & $305.64,316.06,306.06,309.85$ \\
\hline BEBXII & 305.82, 318.84, 306.46, 311.09 \\
\hline FBBXII & $304.26,317.31,304.89,309.56$ \\
\hline FKwBXII & $305.50,318.55,306.14,310.80$ \\
\hline ZBBXII & $302.96,310.78,303.21,306.13$ \\
\hline PBXGL & $290.41,300.83,290.83,294.63$ \\
\hline
\end{tabular}


Table 5: MLEs, SEs and (CIs) for the data set II.

\begin{tabular}{|c|c|}
\hline Model & Estimates \\
\hline$\overline{\mathrm{BXII}(\alpha, \beta)}$ & $\begin{array}{c}3.102,0.465 \\
(0.538),(0.077) \\
(2.05,4.16),(0.31,0.62)\end{array}$ \\
\hline $\operatorname{MOBXII}(\alpha, \beta, \gamma)$ & $\begin{array}{c}2.259,1.533,6.760 \\
(0.864),(0.907),(4.587) \\
(0.57,3.95),(0,3.31),(0,15.75)\end{array}$ \\
\hline $\operatorname{TLBXII}(\alpha, \beta, \gamma)$ & $\begin{array}{c}2.393,0.458,1.796 \\
(0.907),(0.244),(0.915) \\
(0.62,4.17),(0,0.94),(0.002,3.59)\end{array}$ \\
\hline$K w B X I I(a, \delta, \alpha, \beta)$ & $\begin{array}{c}14.105,7.424,0.525,2.274 \\
(10.805),(11.850),(0.279),(0.990) \\
(0,35.28),(0.30 .65),(0,1.07),(0.33,4.21)\end{array}$ \\
\hline $\operatorname{BBXII}(a, \delta, \alpha, \beta)$ & $\begin{array}{c}2.555,6.058,1.800,0.294 \\
(1.859),(10.391),(0.955),(0.466) \\
(0,6.28),(0,26.42),(0,3.67),(0,1.21)\end{array}$ \\
\hline $\operatorname{BEBXII}(a, \delta, \alpha, \beta, \gamma)$ & $\begin{array}{c}1.876,2.991,1.780,1.341,0.572 \\
(0.094),(1.731),(0.702),(0.816),(0.325) \\
(1.7,2.06),(0,6.4),(0.40,3.2),(0,2.9),(0,1.21)\end{array}$ \\
\hline $\operatorname{FBBXII}(a, \delta, \alpha, \beta, \gamma)$ & $\begin{array}{c}0.621,0.549,3.838,1.381,1.665 \\
(0.541),(1.011),(2.785),(2.312),(0.436) \\
(0,1.7),(0,2.5), \quad(0,9.3),(0,5.9),(0.8,4.5)\end{array}$ \\
\hline $\operatorname{FKwBXII}(a, \delta, \alpha, \beta, \gamma)$ & $\begin{array}{c}0.558,0.308,3.999,2.131,1.475 \\
(0.442),(0.314),(2.082),(1.833),(0.361) \\
(0,1.4),(0,0.9),(0,3.1),(0,5.7),(0.76,2.2)\end{array}$ \\
\hline $\operatorname{PBXGL}(a, \delta, \theta, \gamma)$ & $\begin{array}{c}3.0514,2.188,0.369,0.65 \\
(3.51),(0.98),(0.21),(0.29) \\
(0,10.05),(0.24,4.16),(0,0.78),(0.07,1.23)\end{array}$ \\
\hline
\end{tabular}




\begin{tabular}{c|c}
\hline \multicolumn{2}{c}{ Table 6: AIC, BIC, CAIC and HQIC values for the data set II. } \\
\hline Model & AIC, BIC, CAIC, HQIC \\
\hline \hline BXII & $209.60,214.15,209.77,211.40$ \\
MOBXII & $209.74,216.56,210.09,212.44$ \\
TLBXII & $211.80,218.63,212.15,214.52$ \\
KwBXII & $208.76,217.86,209.36,212.38$ \\
BBXII & $210.44,219.54,211.03,214.06$ \\
BEBXII & $212.10,223.50,213.00,216.60$ \\
FBBXII & $206.80,218.20,207.71,211.30$ \\
FKwBXII & $206.50,217.90,207.41,211.00$ \\
PBXGL & $\mathbf{2 0 5 . 5 2 , 2 1 4 . 6 2 , 2 0 6 . 1 1 , 2 0 9 . 1 4}$ \\
\hline
\end{tabular}


Table 7: MLEs, SEs and (CIs) for the the data set III.

\begin{tabular}{|c|c|}
\hline Model & Estimates \\
\hline$\overline{\mathrm{BXII}(\alpha, \beta)}$ & $\begin{array}{c}5.615,0.072 \\
(15.048),(0.194) \\
(0,35.11),(0,0.45)\end{array}$ \\
\hline $\operatorname{MOBXII}(\alpha, \beta, \gamma)$ & $\begin{array}{c}8.017,0.419,70.359 \\
(22.083),(0.312),(63.831) \\
(0,51.29),(0,1.03),(0,195.47)\end{array}$ \\
\hline $\operatorname{TLBXII}(\alpha, \beta, \gamma)$ & $\begin{array}{c}91.320,0.012,141.073 \\
(15.071),(0.002),(70.028) \\
(61.78,120.86)(0.008,0.02)(3.82,278.33)\end{array}$ \\
\hline$K w B X I I(a, \delta, \alpha, \beta)$ & $\begin{array}{c}18.130,6.857,10.694,0.081 \\
(3.689),(1.035),(1.166),(0.012) \\
(10.89,25.36),(4.83,8.89),(8.41,12.98),(0.06,0.10)\end{array}$ \\
\hline $\operatorname{BBXII}(a, \delta, \alpha, \beta)$ & $\begin{array}{c}26.725,9.756,27.364,0.020 \\
(9.465),(2.781),(12.351),(0.007) \\
(8.17,45.27),(4.31,15.21),(3.16,51.57),(0.006,0.03)\end{array}$ \\
\hline $\operatorname{BEBXII}(a, \delta, \alpha, \beta, \gamma)$ & $\begin{array}{c}2.924,2.911,3.270,12.486,0.371 \\
(0.564),(0.549),(1.251),(6.938),(0.788) \\
(1.82,4.03),(1.83,3.99),(0.82,5.72),(0,26.08),(0,1.92)\end{array}$ \\
\hline $\operatorname{FBBXII}(a, \delta, \alpha, \beta, \gamma)$ & $\begin{array}{c}30.441,0.584,1.089,5.166,7.862 \\
(91.745),(1.064),(1.021),(8.268),(15.036) \\
(0,210.26),(0,2.67),(0,3.09),(0,21.37),(0,37.33)\end{array}$ \\
\hline $\operatorname{FKwBXII}(a, \delta, \alpha, \beta, \gamma)$ & $\begin{array}{c}12.878,1.225,1.665,1.411,3.732 \\
(3.442),(0.131),(0.034),(0.088),(1.172) \\
(6.13,19.62),(0.97,1.48),(1.56,1.73),(1.24,1.58),(1.43,6.03)\end{array}$ \\
\hline $\operatorname{PBXGL}(a, \delta, \theta, \gamma)$ & $\begin{array}{c}-11.135,35.442,0.052,0.16 \\
(7.822),(41.1),(0.023),(0.04) \\
(-26.7,4.5),(0,117.6),(0.1,0.09),(0.08,0.24) \\
\end{array}$ \\
\hline
\end{tabular}


Table 8: AIC, BIC, CAIC and HQIC values for the data set IV.

\begin{tabular}{c|c}
\hline Model & AIC, BIC, CAIC, HQIC \\
\hline \hline BXII & $518.46,522.62,518.67,520.08$ \\
MOBXII & $387.22,389.38,387.66,389.68$ \\
TLBXII & $385.94,392.18,386.38,388.40$ \\
KwBXII & $385.58,393.90,386.32,388.86$ \\
BBXII & $385.56,394.10,386.30,389.10$ \\
BEBXII & $387.04,397.42,388.17,391.09$ \\
FBBXII & $386.74,397.14,387.87,390.84$ \\
FKwBXII & $386.96,397.36,388.09,391.06$ \\
PBXGL & $\mathbf{3 8 4 . 9 1 , ~ 3 9 3 . 2 2 , ~ 3 8 5 . 6 5 , 3 8 8 . 1 6}$ \\
\hline \hline
\end{tabular}


Table 9: MLEs, SEs and (CIs) for the data set IV.

\begin{tabular}{|c|c|}
\hline Model & Estimates \\
\hline $\operatorname{BXII}(\alpha, \beta)$ & $\begin{array}{c}58.711,0.006 \\
(42.382),(0.004) \\
(0,141.78),(0,0.01)\end{array}$ \\
\hline $\operatorname{MOBXII}(\alpha, \beta, \gamma)$ & $\begin{array}{c}11.838,0.078,12.251 \\
(4.368),(0.013),(7.770) \\
(0,141.78),(0,0.01),(0,27.48)\end{array}$ \\
\hline $\operatorname{TLBXII}(\alpha, \beta, \gamma)$ & $\begin{array}{c}0.281,1.882,50.215 \\
(0.288),(2.402),(176.50) \\
(0,0.85),(0,6.59),(0,396.16)\end{array}$ \\
\hline$K w B X I I(a, \delta, \alpha, \beta)$ & $\begin{array}{c}9.201,36.428,0.242,0.941 \\
(10.060),(35.650),(0.167),(1.045) \\
(0,28.912),(0,106.30),(0,0.57),(0,2.99)\end{array}$ \\
\hline $\operatorname{BBXII}(a, \delta, \alpha, \beta)$ & $\begin{array}{c}96.104,52.121,0.104,1.227 \\
(41.201),(33.490),(0.023),(0.326) \\
(15.4,176.8),(0,117.8),(0.6,0.15),(0.59,1.9)\end{array}$ \\
\hline $\operatorname{BEBXII}(a, \delta, \alpha, \beta, \gamma)$ & $\begin{array}{c}0.087,5.007,1.561,31.270,0.318 \\
(0.077),(3.851),(0.012),(12.940),(0.034) \\
(0,0.3),(0,12.6),(1.5,1.6),(5.9,56.6),(0.3,0.4)\end{array}$ \\
\hline $\operatorname{FBBXII}(a, \delta, \alpha, \beta, \gamma)$ & $\begin{array}{c}15.194,32.048,0.233,0.581,21.855 \\
(11.58),(9.867),(0.091),(0.067),(35.548) \\
(0,37.8),(12.7,51.4),(0.05,0.4),(0.45,0.7),(0,91.5)\end{array}$ \\
\hline $\operatorname{FKwBXII}(a, \delta, \alpha, \beta, \gamma)$ & $\begin{array}{c}14.732,15.285,0.293,0.839,0.034 \\
(12.390),(18.868),(0.215),(0.854),(0.075) \\
(0,39.02),(0,52.27),(0,0.71),(0,2.51),(0,0.18)\end{array}$ \\
\hline $\operatorname{ZBBXII}(a, \alpha, \beta)$ & $\begin{array}{c}41.973,0.157,44.263 \\
(38.787),(0.082),(47.648) \\
(0,117.99),(0,0.32,)(0,137.65)\end{array}$ \\
\hline $\operatorname{PBXGL}(a, \delta, \theta, \gamma)$ & $\begin{array}{c}-5.1,0.035,0.62,12.6 \\
(0.00),(0.00),(0.03), \quad(0.00) \\
-,-,(0.65,0.68),-\end{array}$ \\
\hline
\end{tabular}


Table 10: AIC, BIC, CAIC and HQIC values for the data set IV.

\begin{tabular}{c|c}
\hline Model & AIC, BIC, CAIC, HQIC \\
\hline \hline BXII & $328.20,331.19,328.60,329.19$ \\
MOBXII & $315.54,320.01,316.37,317.04$ \\
TLBXII & $316.26,320.73,317.09,317.76$ \\
KwBXII & $317.36,323.30,318.79,319.34$ \\
BBXII & $316.46,322.45,317.89,318.47$ \\
BEBXII & $317.58,325.06,319.80,320.09$ \\
FBBXII & $317.86,325.34,320.08,320.36$ \\
FKwBXII & $317.76,325.21,319.98,320.26$ \\
ZBBXII & $313.86,318.35,314.39,315.36$ \\
PBXGL & $\mathbf{3 1 1 . 2 3 , 3 1 7 . 2 2 , 3 1 2 . 6 6 , 3 1 3 . 2 5}$ \\
\hline \hline
\end{tabular}


Estimated CDF (Breaking Stress Data)

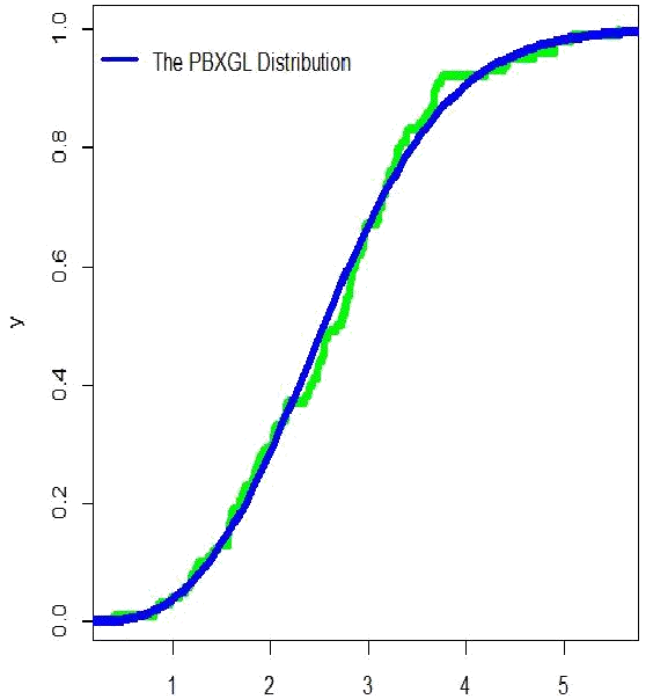

Data I

Estimated CDF (Taxes Revenue Data)

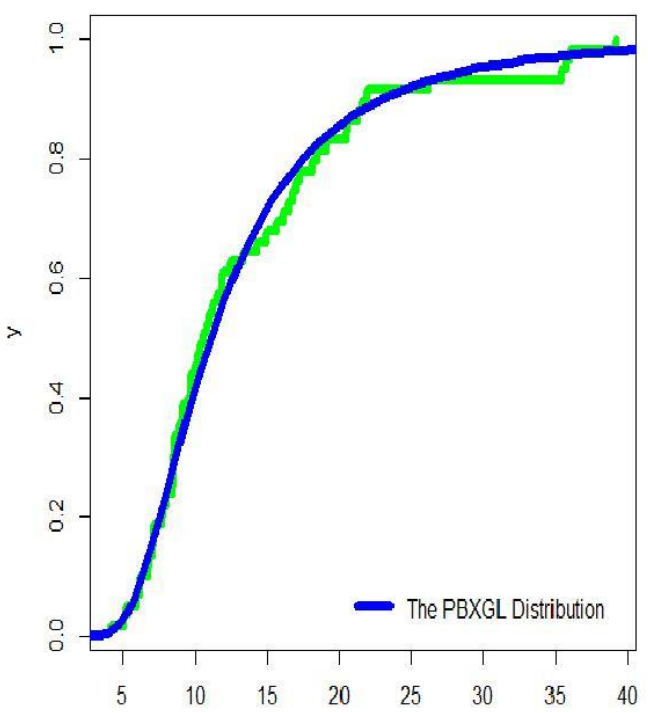

Estimated CDF (Survival Times Data)

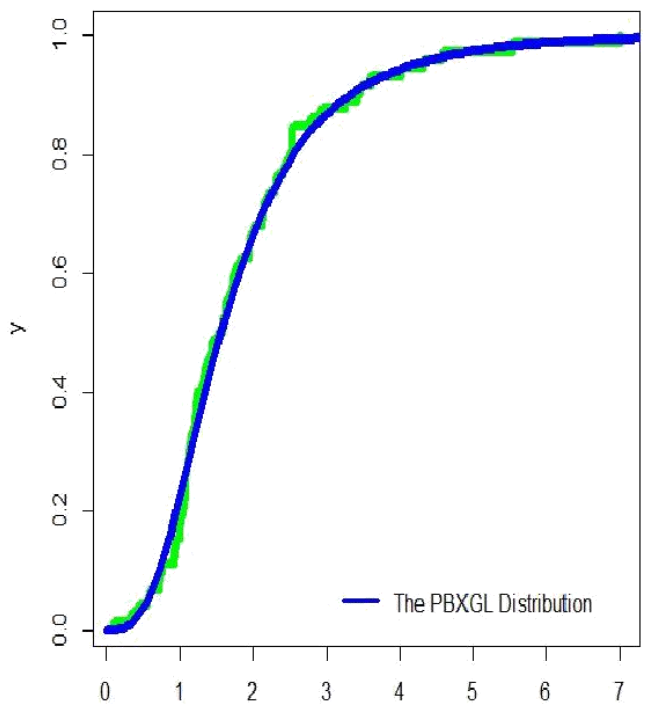

Data II

Estimated CDF (leukemia Data)

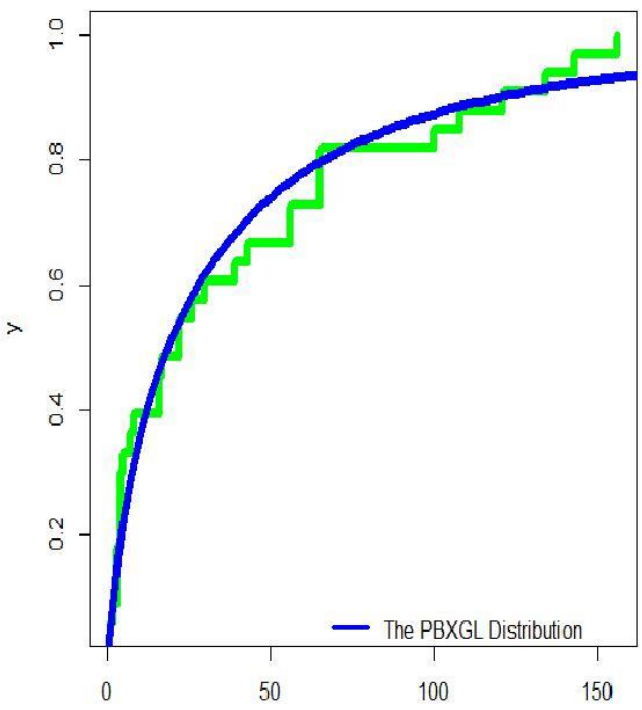

Data III

Data IV

Figure 4: Estimated CDFs. 


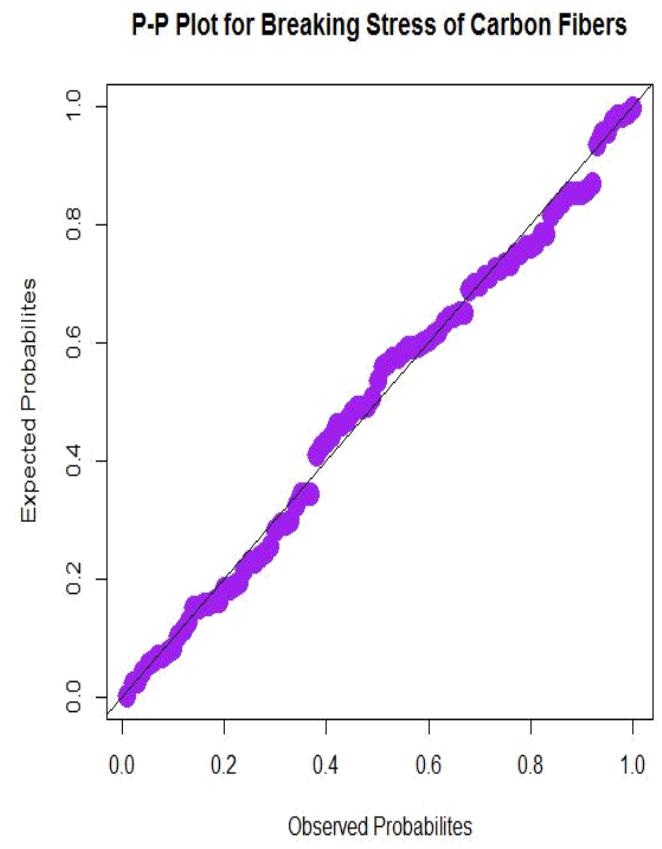

Data I

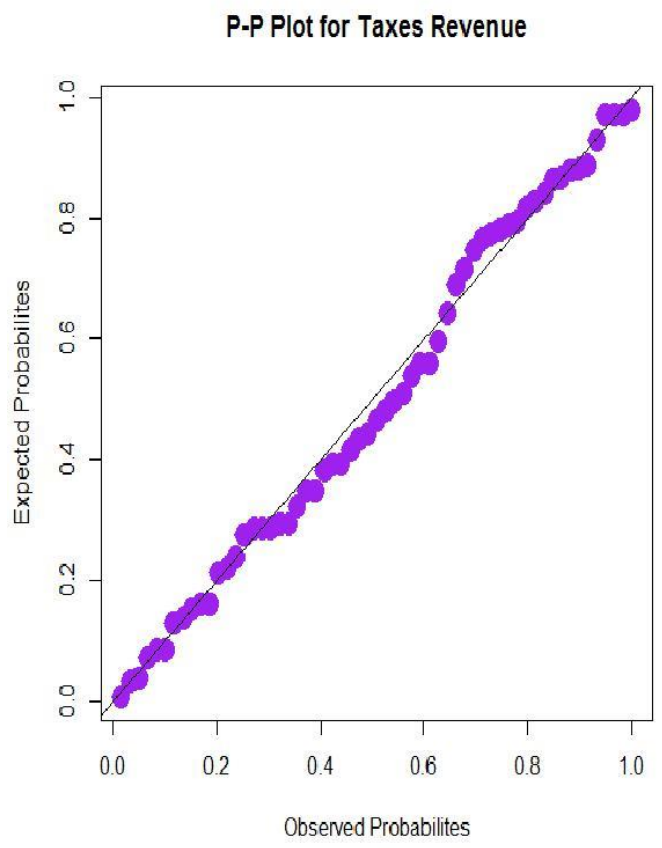

Data III

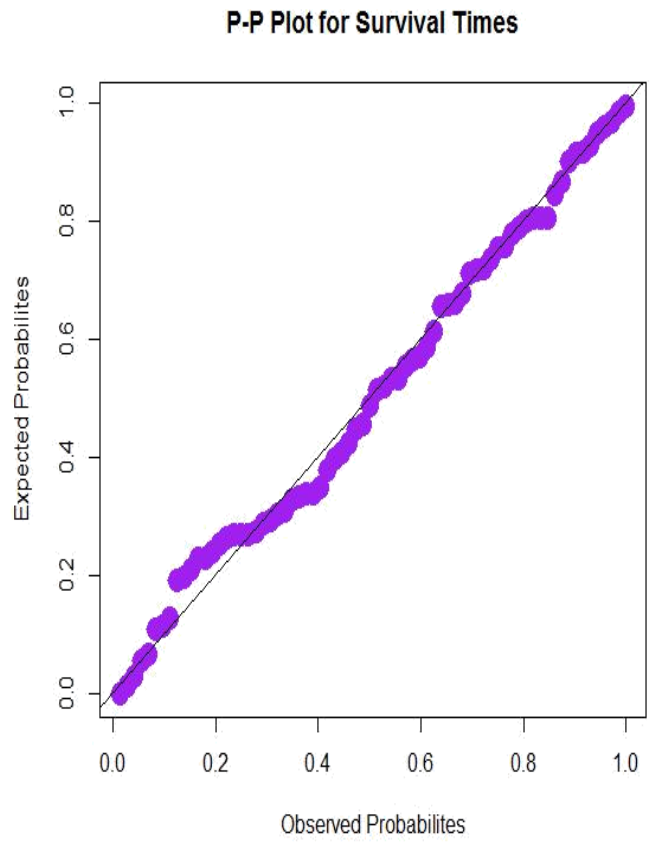

Data II

P.P Plot for Survival Times (Myelogeneous leukemia)

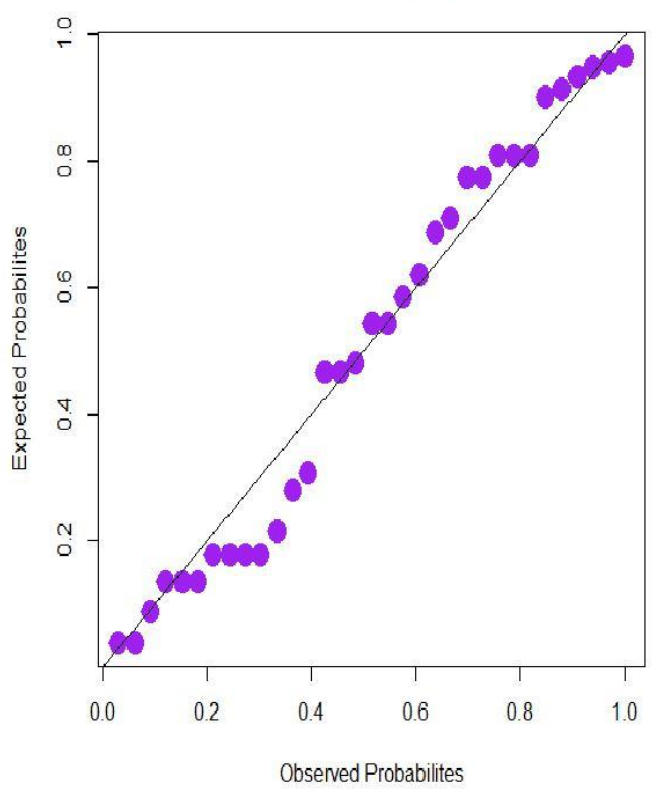

Data IV

Figure 5: P-P plots. 
Kaplan-Meier Survival Plot

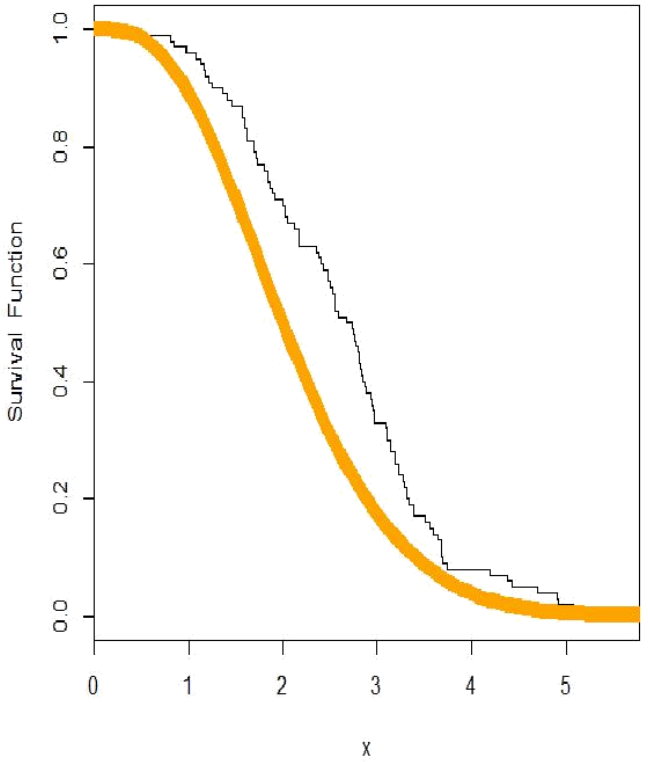

Data I

Kaplan-Meier Survival Plot

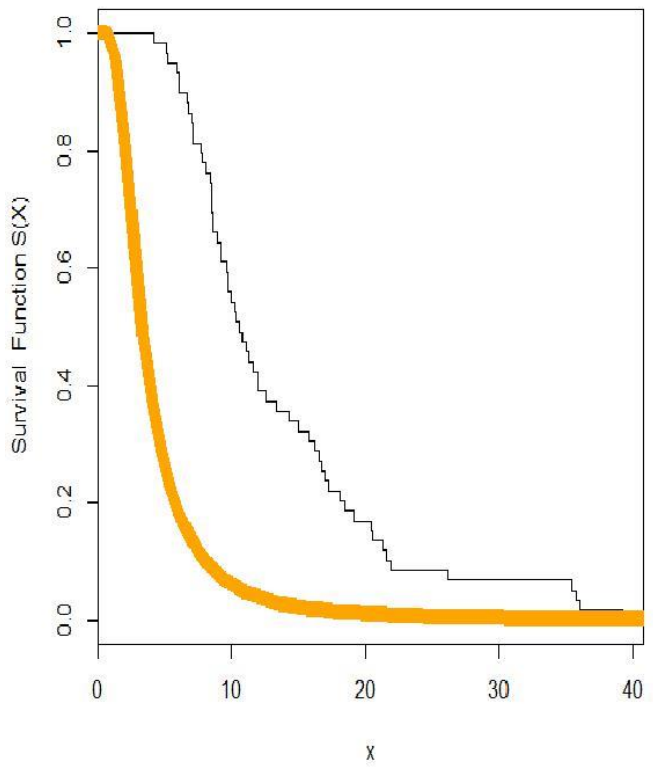

Data III
Kaplan-Meier Survival Plot

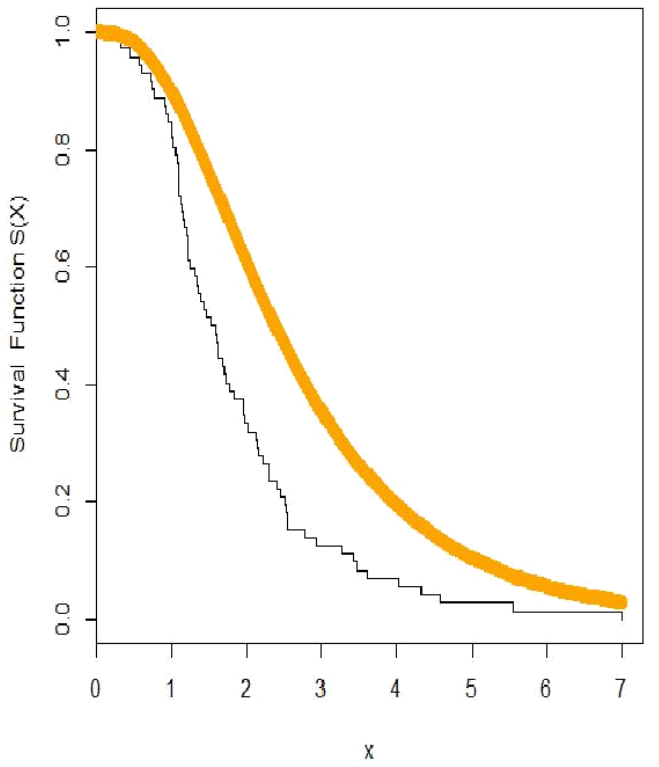

Data II

Kaplan-Meier Survival Plot

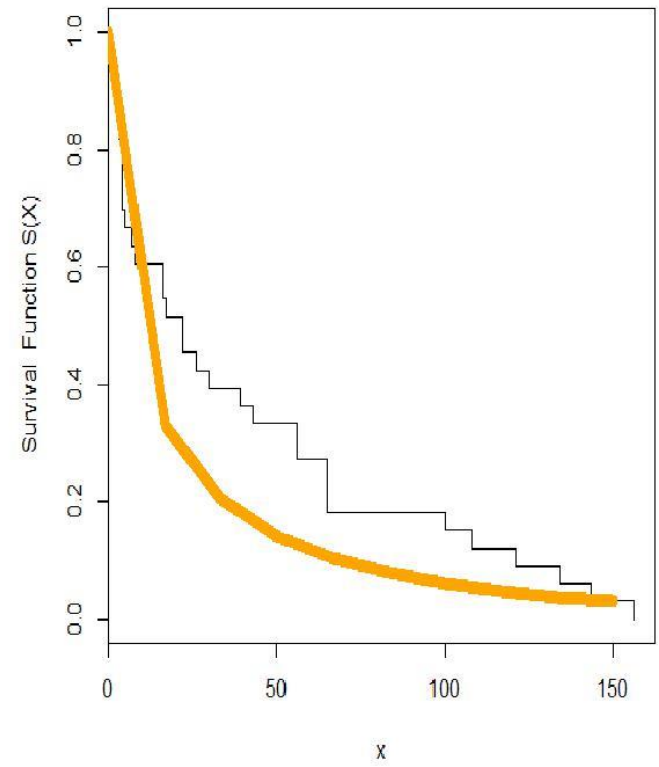

Data IV

Figure 6: Kaplan-Meier survival plots. 


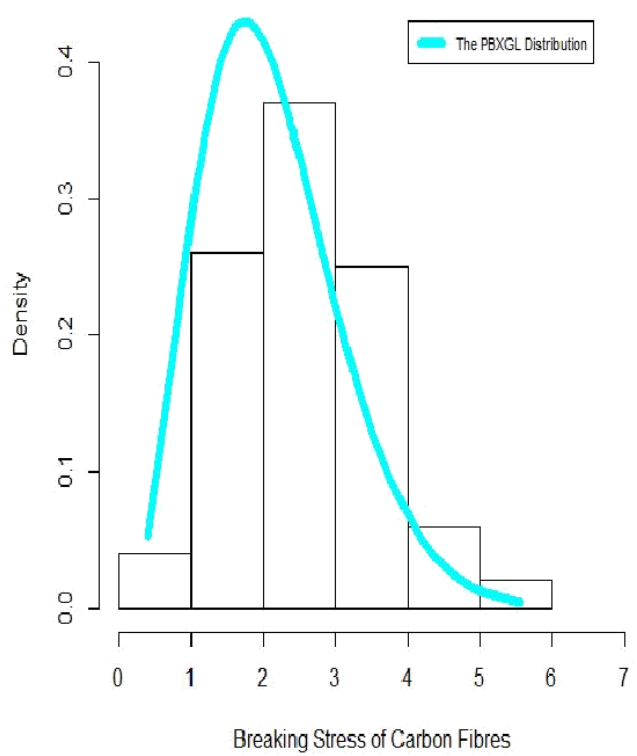

Data I

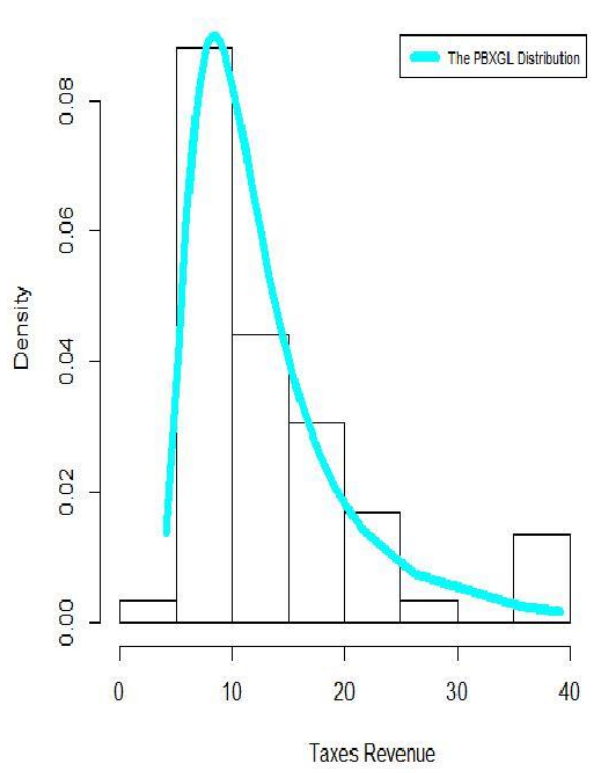

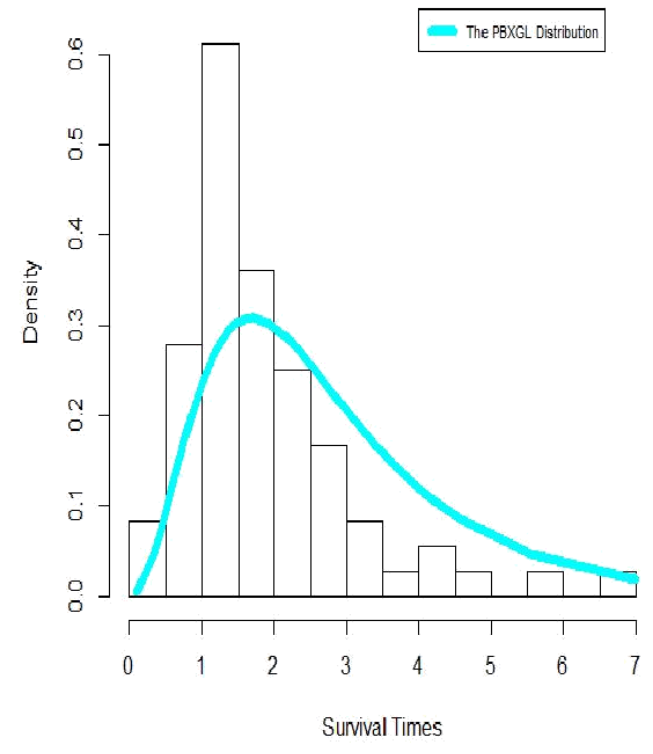

Data II

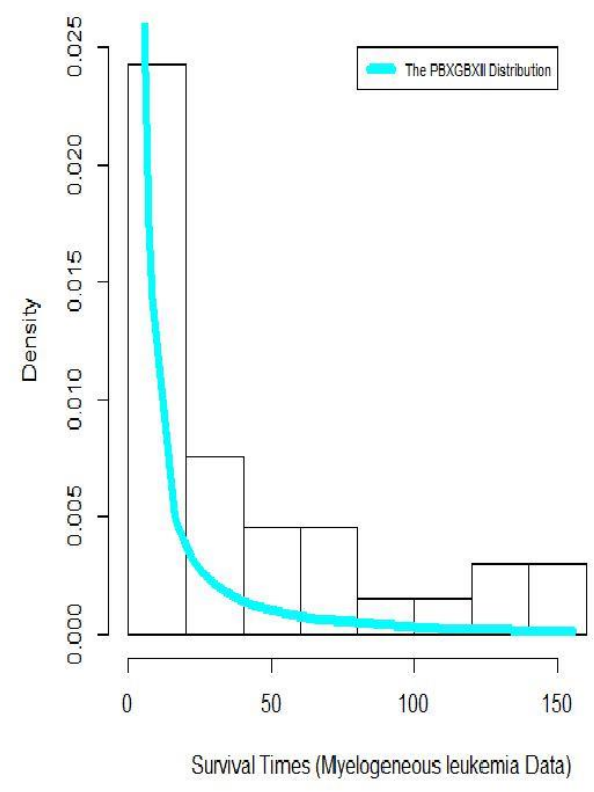

Data IV

Data III

Figure 7: Estimated PDFs. 


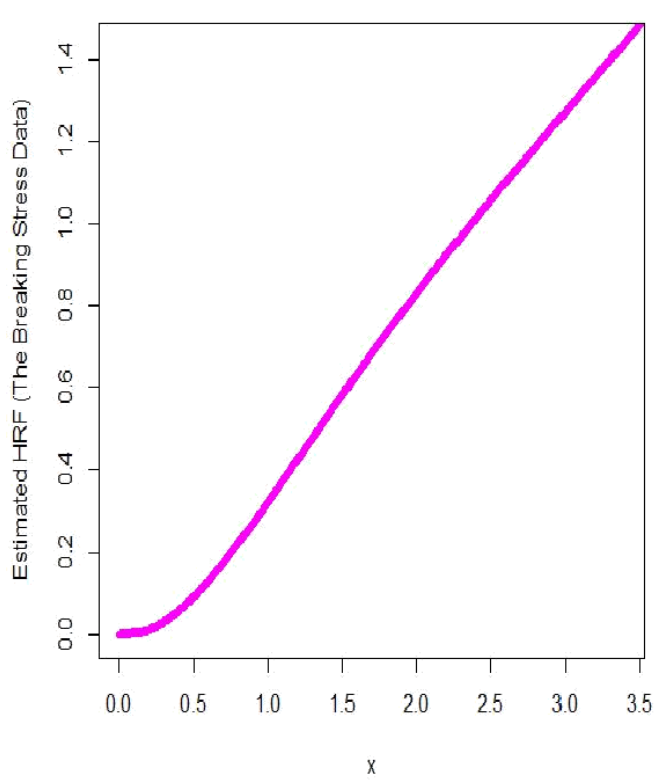

Data I

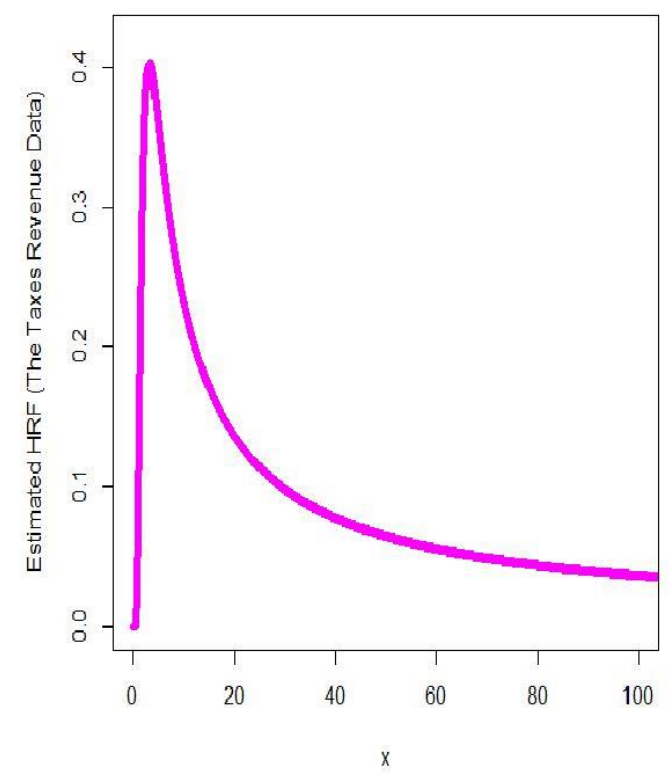

Data III

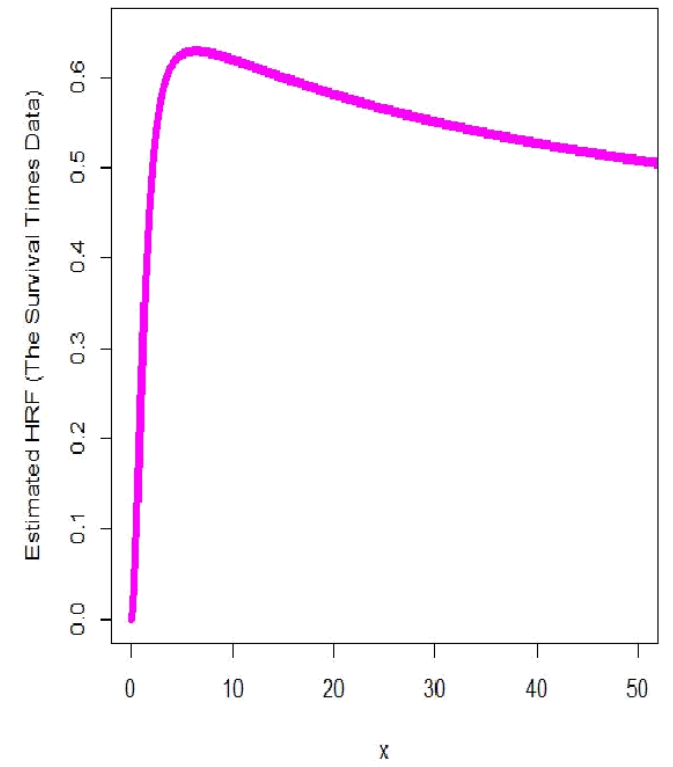

Data II

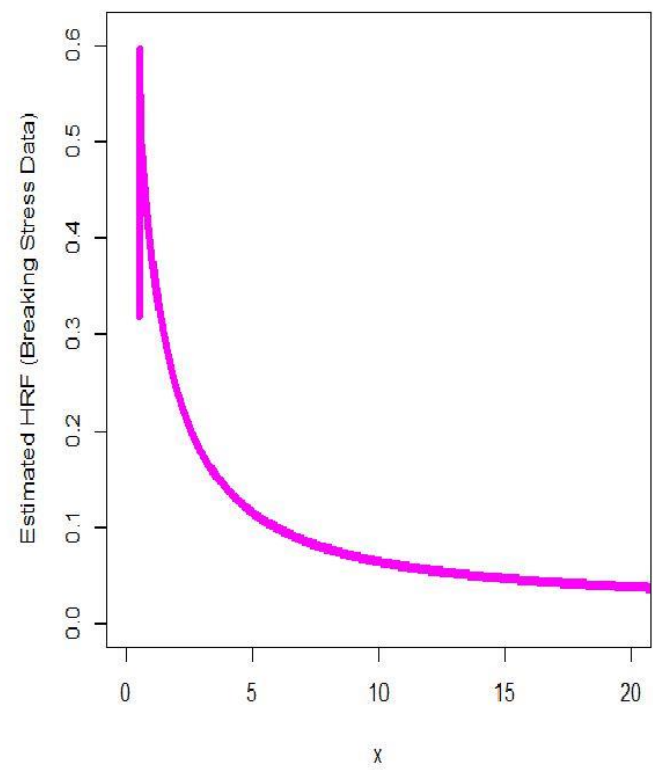

Data IV

Figure 8: Estimated HRFs.

\section{Conclusions}

A new version of the Poisson Lomax distributions called the Poisson Burr X generalized Lomax (PBXGL) is proposed and studied. The new density function is expressed as a linear mixture of the Lomax densities. The PBXGL density can be right skewed and unimodal. The 
failure rate function of the PBXGL model can be increasing-constant or increasing or U shape or decreasing or upside down-increasing. We are also motivated to introduce the PBXGL distribution that contains many new lifetime models. The statistical properties are derived and four applications are provided to illustrate the importance of the new PBXGL density. The method of maximum likelihood is used to estimate the unknown parameters of the PBXGL density. Adequate fitting is provided by the new model.

\section{Acknowledgment}

The authors gratefully acknowledge with thanks the very thoughtful and constructive comments and suggestions of the Editor-in-Chief and the reviewers which resulted in much improved paper.

\section{References}

[1] Aarset, M. V. (1987). How to identify a bathtub hazard rate. IEEE Transactions on Reliability, 36(1), 106-108.

[2] Altun, E., Yousof, H. M. and Hamedani G. G. (2018 a). A new log-location regression model with influence diagnostics and residual analysis. International Journal of Applied Mathematics and Statistics, forthcoming.

[3] Altun, E., Yousof, H. M., Chakraborty, S. and Handique, L. (2018 b). Zografos-Balakrishnan Burr XII distribution: regression modeling and applications. International Journal of Mathematics and Statistics, forthcoming.

[4] Bjerkedal, T. (1960). Acquisition of resistance in Guinea pigs infected with different doses of virulent tubercle bacilli. American Journal of Hygiene, 72, 130-148.

[5] Brito, E., Cordeiro, G. M., Yousof, H. M., Alizadeh, M. and Silva, G. O. (2017). Topp-Leone Odd Log-Logistic Family of Distributions, Journal of Statistical Computation and Simulation, 87(15), 3040-3058.

[6] Burr, I. W. (1942). Cumulative frequency functions. Annals of Mathematical Statistics, 13, 215-232.

[7] Burr, I. W. (1968). On a general system of distributions, III. The simplerange. Journal of the American Statistical Association, 63, 636-643.

[8] Burr, I. W. (1973). Parameters for a general system of distributions to match a grid of 3 and 4. Communications in Statistics, 2, 1-21.

[9] Burr, I. W. and Cislak, P. J. (1968). On a general system of distributions: I. Its curve-shaped characteristics; II. The sample median. Journal of the American Statistical Association, 63, 627-635.

[10] Cordeiro, G. M., Yousof, H. M., Ramires, T. G. and Ortega, E. M. M. (2018). The Burr XII system of densities: properties, regression model and applications. Journal of Statistical Computation and Simulation, 88(3), 432-456.

[11] Elbiely, M. M. and Yousof, H. M. (2019). A new flexible Weibull Burr XII distribution. Journal of Statistics and Applications, 2(1), 59-77. 
[12] Feigl, P., and Zelen, M. (1965). Estimation of Exponential Survival Probabilities with Concomitant Information, Biometrics, 21, 826-838, www.jstor.org/stable/2528247.

[13] Gad, A. M., Hamedani, G. G., Salehabadi, S. M. and Yousof, H. M. (2019). The Burr XII-Burr XII distribution: mathematical properties and characterizations. Pakistan Journal of Statistics, 35(3), 229-248.

[14] Gradshteyn, I. S. and Ryzhik, I. M. (2000). Table of Integrals, Series and Products (sixth edition). San Diego: Academic Press.

[15] Goual, H. and Yousof, H. M. (2019). Validation of Burr XII inverse Rayleigh model via a modified chi-squared goodness-of-fit test. Journal of Applied Statistics, 47(1), 1-32.

[16] Goual, H., Yousof, H. M. and Ali, M. M. (2019). Validation of the odd Lindley exponentiated exponential by a modified goodness of fit test with applications to censored and complete data. Pak. J. Stat. Oper. Res. 15(3), 745-771.

[17] Hamedani G. G., Altun, E, Korkmaz, M. C., Yousof, H. M. and Butt, N. S. (2018). A new extended $\mathrm{G}$ family of continuous distributions with mathematical properties, characterizations and regression modeling. Pak. J. Stat. Oper. Res., 14(3), 737-758.

[18] Hamedani G. G. Rasekhi, M., Najibi, S. M., Yousof, H. M. and Alizadeh, M., (2019). Type II general exponential class of distributions. Pak. J. Stat. Oper. Res., XV(2), 503-523.

[19] Hamedani G. G. Yousof, H. M., Rasekhi, M., Alizadeh, M., Najibi, S. M. (2017). Type I general exponential class of distributions. Pak. J. Stat. Oper. Res., XIV(1), 39-55.

[20] Ibrahim, M. (2019). The compound Poisson Rayleigh Burr XII Distribution: Properties and Applications. Journal of Applied Probability and Statistics, forthcoming.

[21] Korkmaz, M. C. Yousof, H. M., Rasekhi, M. and Hamedani G. G. (2018). Bayesian analysis, classical inference and characterizations for the odd Lindley Burr XII model. Mathematics and Computers in Simulation. Journal of Data Science, 16(2), 327-354.

[22] Nichols, M.D. and Padgett, W.J. (2006). A bootstrap control chart for Weibull percentiles. Quality and Reliability Engineering International, 22, 141-151.

[23] Paranaíba, P. F. P., Ortega, E. M. M., Cordeiro, G. M. and Pescim, R. R. (2011). The beta Burr XII distribution with application to lifetime data. Computation Statistics and Data Analysis, 55, 11181136 .

[24] Prudnikov, A. P., Brychkov, Y. A. and Marichev, O. I. (1986). Integrals and Series, 1. Gordon and Breach Science Publishers, Amsterdam.

[25] Prudnikov, A. P., Brychkov, Y. A. and Marichev, O. I. (1992). Integrals and Series, 4. Gordon and Breach Science Publishers, Amsterdam.

[26] Rodriguez, R.N. (1977). A guide to the Burr type XII distributions. Biometrika, 64, 129-134.

[27] Tadikamalla, P. R. (1980). A look at the Burr and related distributions. International Statistical Review, 48, 337-344.

[28] Yousof, H. M., Afify, A. Z., Hamedani, G. G. and Aryal, G. (2017). The Burr X generator of distributions for lifetime data. Journal of Statistical Theory and Applications, 16, 288-305. 
[29] Yousof, H. M., Ahsanullah, M. and Khalil, M. G. (2019 a). A new zero-truncated version of the Poisson Burr XII distribution: characterizations and properties. Journal of Statistical Theory and Applications, 18(1), 1-11.

[30] Yousof, H. M., Altun, E., Ramires, T. G., Alizadeh, M. and Rasekhi, M. (2018). A new family of distributions with properties, regression models and applications. Journal of Statistics and Management Systems, 21, 163-188.

[31] Yousof, H. M., Rasekhi, M., Altun, E., Alizadeh, M. Hamedani G. G. and Ali M. M. (2019 b). A new lifetime model with regression models, characterizations and applications. Communications in Statistics-Simulation and Computation, 48(1), 264-286. 